\title{
Citrus Pest Quick Guide: Citrus Leafminer (Phyllocnistis citrella Stainton)
}

\section{Life Cycle}

Eggs look like tiny dew drops on young expanding leaf growth. Eggs are usually found beside the midvein on the underside of an unexpanded leaf.

Larvae emerge directly into leaf tissue and begin mining along the midvein in a back and forth pattern until pupation occurs. If populations are high, several larvae can be found in one leaf and even on young stems.

Adults are small moths with a $4 \mathrm{~mm}$ wingspan and can resemble a speck of dust. They shelter in the tree canopy during the day and emerge at night to deposit eggs. Females can lay 50 eggs in their short lifespan, causing populations to grow quickly.

\section{Damage}

Larval feeding causes distorted leaves with reduced photosynthetic capacity. Full leaf expansion is often not completed. Due to the damage, there is chlorosis on the other side of the leaf. In severe cases, leaf drop and possible stem dieback can occur.

Damage generally starts in spring as temperatures rise and can increase rapidly under ideal conditions. The lowest populations are in the winter months, and the highest populations are in spring and summer. Population levels vary from year to year.

Leafminer damage also becomes an entry point for citrus canker bacteria.

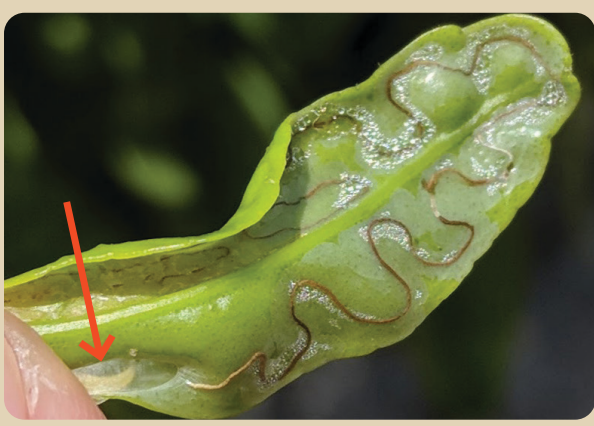

Citrus leafminer larva.

Credit: UF/IFAS

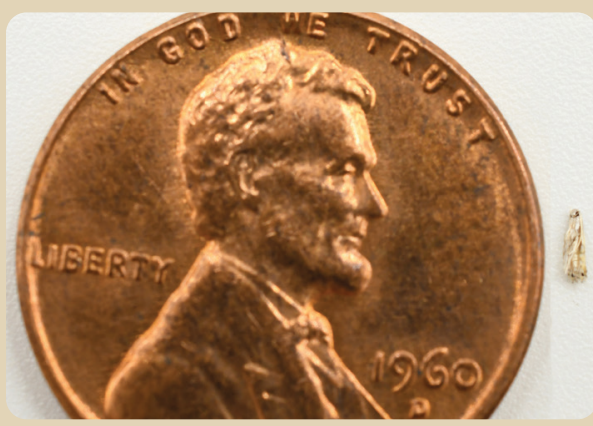

Citrus leafminer adult (right of penny). Credit: T. R. Weeks, UF/IFAS

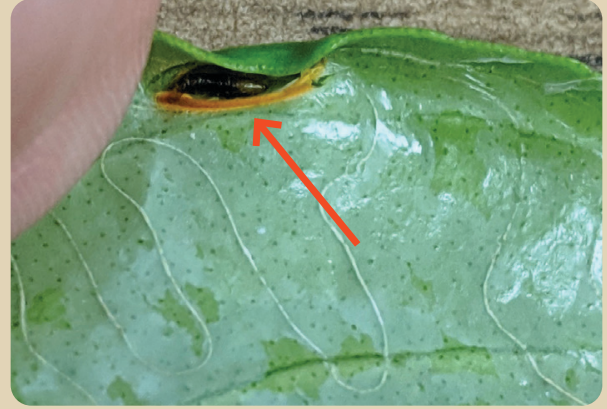

Citrus leafminer pupa.

Credit: L. M. Diepenbrock, UF/IFAS

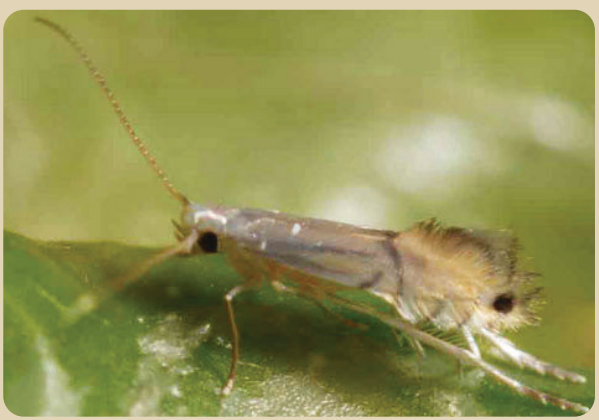

Citrus leafminer adult magnified. Credit: L. Buss, UF/IFAS
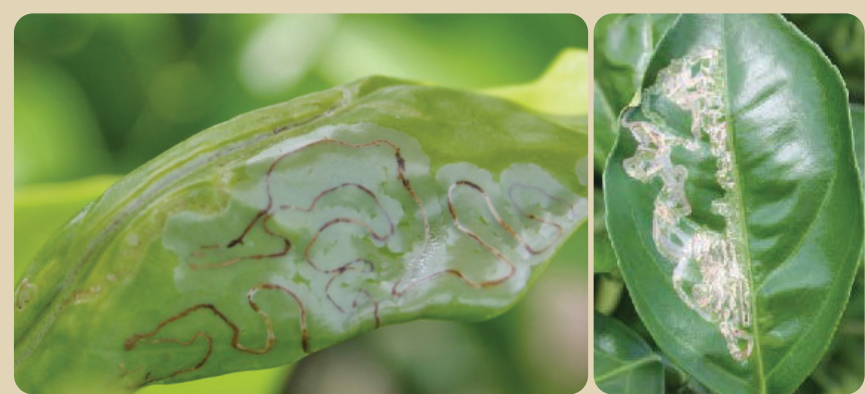

Citrus leafminer damage.

Credit: J. D. Burrow, UF/IFAS

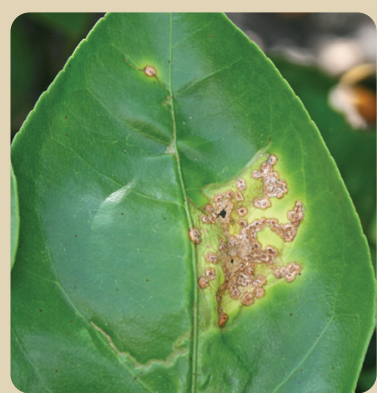

Citrus leafminer damage with canker lesions. Credit: J. D. Burrow, UF/IFAS 